\title{
Status of the PADME experiment and review of dark photon searches
}

\author{
M. Raggi ${ }^{1}$ \\ ${ }^{1}$ Sapienza Università di Roma ed INFN, Sezione di Roma \\ P.le A. Moro 2, I-00185 Roma (Italy). \\ e-mail: mauro.raggi@roma1.infn.it
}

\begin{abstract}
While accelerator particle physics has traditionally focused on exploring dark matter through highenergy experiments, testing dark-sectors hypothesis requires innovative low energy experiments that use highintensity beams and high-sensitivity detectors. In this scenario attractive opportunities are offered to low energy machines and flavour experiments. In this paper we will focus our attention on the Dark Photon (DP) scenario, reviewing the current status of searches and new opportunities with particular attention to the PADME experiment at Laboratori Nazionali di Frascati.
\end{abstract}

\section{Introduction}

The existing experimental program to search for dark matter is focused primarily on weakly-interacting massive particles (WIMPs). At the same time, given the importance of dark matter, there are strong motivations to explore a broader set of dark matter candidates. Recent null results obtained in Dark Matter candidates searches at the Large Hadron Collider, together with the increasing sensitivity reached in direct detection without any evidence of WIMPs, have revitalised the idea that dark matter can have sub-GeV mass. In addition recent anomalies in the magnetic momentum of the muon, in the decay of ${ }^{8} \mathrm{Be}$, and proton radius can be solved introducing light new states[1]. A simple possibility is that dark matter interacts through a new force that is similar in structure to the known forces, but couples only indirectly to ordinary matter through new particles called "mediators".

\section{Dark Sector Panorama}

A dark sector is a collection of particles that are not directly charged under the Standard Model (SM) forces. Such particles are assumed to possess gravitational interactions, and may also interact with familiar matter through several "portal" interactions that are constrained by the symmetries of the Standard Model. The dark sector typically include one or more mediator particles coupled to the SM via the portal interaction. The portal relevant for dark sector-SM interactions depends on the mediator spin and parity: it can be a scalar, a pseudo-scalar, a fermion, or a vector.

We will focus on the vector portal, but we will briefly comment on the other possible mediators. If the mediator is a scalar, it can interact via the Higgs portal and can be probed in various ways, including exotic Higgs decays at high-energy colliders such as the LHC. If the mediator is a pseudoscalar a valuable candidate is the pseudo NambuGoldstone boson of the Peccei -Quinn U(1) symmetry: the axion. A possible existence of a sterile neutrino $\mathrm{N}$ may lead to the addition of Yukawa term in the lagrangian requiring a fermionic mediator.

The most general interaction of an electrically neutral vector particle A with the Standard Model fermions can be written in the form of a U(1) interaction. Its mediator will be a massive "photon like" particle which couples to SM with a strength $\epsilon e$. In the present review we focus mostly on the vector portal as this scenario is able produce signatures accessible in flavour and low energy experiments.

\section{Dark Photon Models}

We will call dark photon model every model that predicts the existence of a new neutral vector particle $A^{\prime}$ which has a non-vanishing coupling to the standard fermions. The $A^{\prime}$ could be the mediator between the SM and the dark sector. The origin of the coupling of $A^{\prime}$ to the fermion fields could arise in various models. Since almost any extension of the Standard Model introduces new symmetries and gauge groups, the wide range of possibilities go from maximally universal models to the ones including a single type of fermions or even a single lepton generation[1].

The production of $A^{\prime}$ in collider experiments relies on the DP couplings to SM particles, primarily electrons and quarks. In the simplest scenario the couplings arise from the "kinetic mixing" interaction, which mixes the $A$ ' boson of the non-SM "dark" gauge group $U(1)_{D}$ with the SM photon:

$$
\mathcal{L}=\frac{1}{2} \epsilon F^{\mu v} F_{\mu \nu}^{\prime}
$$


where $\epsilon$ is a dimensionless parameter while $F^{\mu v}$ is the tensor of the SM $U(1)_{e m}$ and $F_{\mu \nu}^{\prime}$ of the dark $U(1)_{D}$ respectively.

Due to the mixing with the standard model photon, the DP can be produced in electromagnetic processes similar to those in the SM. The most considered are: emission of $A^{\prime}$ in electron bremsstrahlung ( $A^{\prime}$-strahlung), $e^{+} e^{-} \rightarrow \gamma A^{\prime}$ annihilation, and mesons decays with photons in the final state $\left(\pi^{0}, \eta \rightarrow \gamma A^{\prime}\right)$.

The DP decay modes depend on its mass and on the hypothesis on the dark sector particles mass spectrum. The most commonly used are to consider the DP the lightest state in the dark sector and that it decays to SM particles, "visible decays". Depending on the $A^{\prime}$ mass di-lepton or hadronic decays are allowed. In more general models the existence of dark fermions $\chi$ with mass $m_{\chi}<m_{A^{\prime}} / 2$, coupling to DP with a strength $\alpha_{D}$, allows the $A^{\prime} \rightarrow \chi \bar{\chi}$ decay channel. In natural scenario, $\alpha_{D}>>\epsilon^{2}$ since there is no necessity to suppress the interactions in the hidden sector, the dominant decay channel would be $A^{\prime} \rightarrow \chi \bar{\chi}$, "invisible decay.".

The two scenarios, so called "visible" and "invisible", produce very different experimental signatures. In the visible case a narrow resonance might be observed in the di-lepton or, in general, in the di-particle invariant mass spectrum, while in the invisible case the DP could manifest itself through the search for missing mass or missing energy. A detailed review of the DP photon physics and experimental bounds can be found in [1] [2].

\subsection{Visible dark photon search status}

In the case of the visible dark photon model with universal coupling to SM fermions, the present experimental situation is summarised in Fig. 1. Experiments searching for bumps in di-lepton invariant mass distributions ruled out $\epsilon$ values greater than $10^{-3}$ in the $10 \mathrm{MeV}-10 \mathrm{GeV}$ mass range. NA48/2 at CERN [3], A1 at Mainz [4], and BaBar at SLAC [5] provide the strongest experimental bounds. These experiments employ different dark photon production mechanisms, including meson decays (NA48/2), electron bremsstrahlung (A1), and $e^{+} e^{-}$to two photon annihilation (BaBar). The region of $\epsilon<10^{-4}$ is out of reach for bump hunt experiments due to standard model background. Beam dump experiments, such as E137[6] at SLAC profit of massive absorbers, beam dumps, to kill the SM background and detect the decay product of long living $A^{\prime}$ traversing the dump without interacting. There are also theoretical constraints derived from the measurement of the anomalous magnetic moment of the electron and muon $a_{e}, a_{\mu}$. All the existing constraints, in the hypothesis of universal coupling to fermions, rule out the possibility that the visible dark photon model can explain the observed anomaly in the magnetic moment of the muon.

\subsection{Invisible dark photon search status}

The parameter space is significantly less constrained in the case of $A^{\prime}$ decaying to dark-sector ("invisible") final

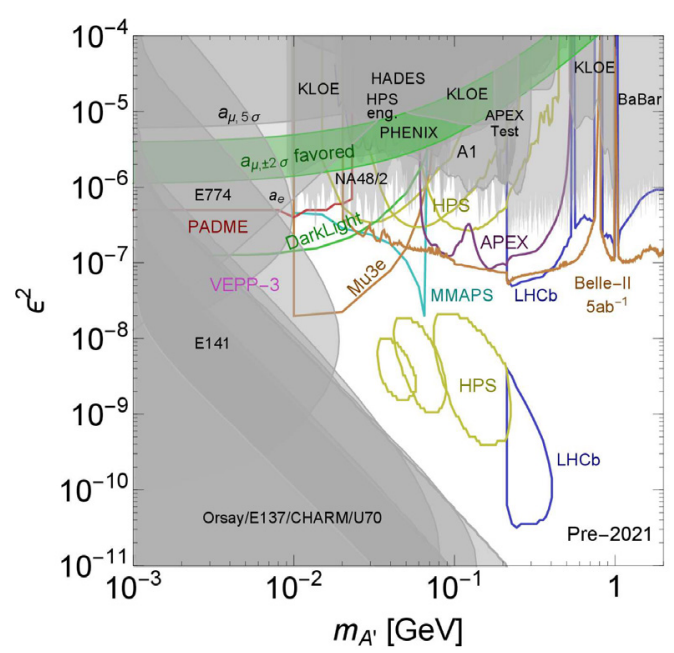

Figure 1. Status of DP visible decay searches [1]. Filled area are already excluded while lines represent projections for future dedicated searches.

states, see Fig.2. In this case, the only bounds come from BaBar[7], using $e^{+} e^{-} \rightarrow \gamma A^{\prime}$, NA64 at CERN[8] using missing energy. The bounds from the $a_{e}$ measurement also applies, since it relies on virtual dark photon contribution and as such is insensitive to the $A^{\prime}$ decay mode. Recently

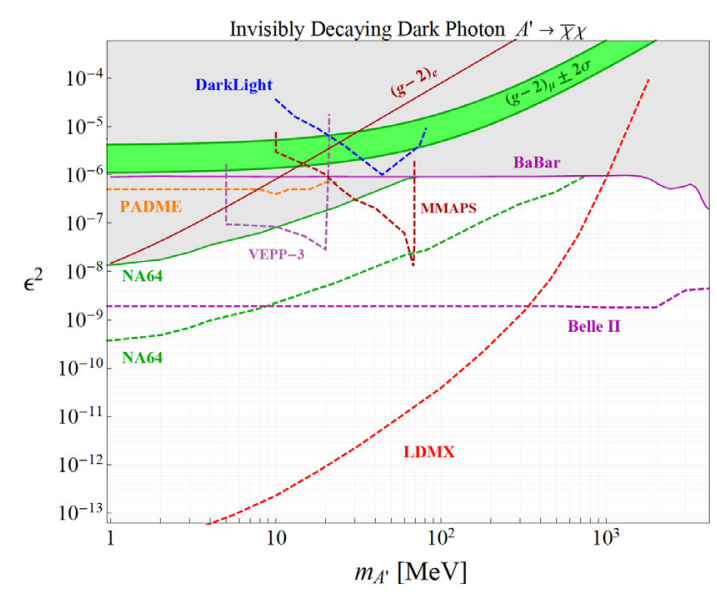

Figure 2. Status of DP invisible decay searches [1]. Filled area are already excluded while dashed lines represent projections for future dedicated searches.

the Babar and NA64 measurements covered the parameter region favoured by the $(g-2)_{\mu}$ anomaly explanation, disfavouring DP as a candidate solution. Additional constraints can be extracted by looking for the scattering dark fermions $(\chi)$, obtained from DP decays, in beam dump experiments. A summary of such a kind of bounds is presented in [1].

\section{Opportunities in Kaon decays}

Kaon physics experiments have a long tradition in precision measurements. Huge samples of Kaon decays have been collected during the last 2 decades allowing NA48/2 
to set already a strong bound in the visible DP parameter space. New possibilities can still be explored with extended mass reach or different production and decay mechanisms.

Kaon radiative decays are a natural place where $A^{\prime}$ can be produced. Since the interaction is mediated by mixing with the photon, the natural place to look for $A^{\prime}$ are the decays of kaons with photon or lepton pairs in the final state. We will consider two important processes:

- $K^{+} \rightarrow \pi^{+} \gamma^{*}$ mixing with $K^{+} \rightarrow \pi^{+} A^{\prime}$

- $K^{+} \rightarrow \ell^{+} v \gamma^{*}$ mixing with $K^{+} \rightarrow \ell^{+} v A^{\prime}$

Modern kaon experiments, in particular NA62[9] and KOTO[10], are able to explore kaon decays with the very high luminosity and precision detectors needed to search for new light weakly coupled particle.

\subsection{The $K^{+} \rightarrow \pi^{+} A^{\prime}$ decay}

An $A^{\prime}$ mixing to virtual photon can be produced in the decay $K^{+} \rightarrow \pi^{+} X$. In the visible dark photon scenario the $A^{\prime}$ will promptly decay in standard model leptons producing, depending on its mass, pairs of electrons or muons. The invariant mass spectrum of $e^{+} e^{-}$or $\mu^{+} \mu^{-}$, in $K^{+} \rightarrow \pi^{+} e^{+} e^{-}$ an $K^{+} \rightarrow \pi^{+} \mu^{+} \mu^{-}$decays respectively, may reveal the presence of a resonance via the bump hunt search. The recent null result by NA48/2 in bump hunt search in the di-muon invariant mass of $K^{+} \rightarrow \pi^{+} \mu^{+} \mu^{-}$decay[11] even if is not competitive with the best limits in the kinetic mixing scenario, might have interesting implications in case of non universal flavour couplings. More stringent limits are in within reach of NA62 for the invisible decay scenario: $K^{+} \rightarrow \pi^{+} A^{\prime}$ with $A^{\prime} \rightarrow \chi \bar{\chi}$. The new physics signature $K^{+} \rightarrow \pi^{+}$missing - energy can only be realised in the SM via the strongly suppressed FCNC process $K^{+} \rightarrow \pi^{+} v \bar{v}$, and therefore even a relatively modest excess of events may lead to a clear discovery. NA62 experiment, currently taking data at CERN SPS, aiming at collecting 100 events of $K^{+} \rightarrow \pi^{+} v \bar{v}$ with very low background contamination, has the possibility to improve strongly the current bound coming from E787-E949 (See Fig.5). Invisibly decaying DP will manifest itself as an anomaly in the Missing Mass distribution of the background in the NA62 data sample.

\subsection{The $K^{+} \rightarrow \ell^{+} v A^{\prime}$ decay}

Rare radiative leptonic decays might reveal signatures of particles that couple predominantly to leptons, avoiding part of the constraints shown in Fig 1. In the $K^{+} \rightarrow$ $\mu^{+} v e^{+} e^{-}$and $K^{+} \rightarrow \mu^{+} v \mu^{+} \mu^{-}$processes, kaons are a source of tagged muons, and the $A^{\prime}$ can be produced even in absence of coupling to quarks. Models have been proposed with light scalars only coupling to muons[12] thus circumventing almost all present bounds. In this scenario an interesting role might be played by $K^{+} \rightarrow \mu^{+} v \mu^{+} \mu^{-}$final state, in which both production, $A^{\prime}$-strahlung from a $\mu$ leg, and the $A^{\prime}$ decay, $A^{\prime} \rightarrow \mu^{+} \mu^{-}$, are allowed even if DP only couples to muons. NA48/2 collaboration is currently exploring the di-electron decay mode [13].

\section{The PADME experiment at LNF}

The PADME experiment [14][15] at the DAФNE LINAC of the INFN Laboratori Nazionali di Frascati (LNF), has been designed to search for the dark photon by using a positron beam hitting a low $\mathrm{Z}$ target. The $A^{\prime}$ can be observed by searching for a narrow unexpected peak in the spectrum of the missing mass measured in single photon final states. The measurement requires the precise determination of the 4-momentum of the recoil photon and of the impinging positron.

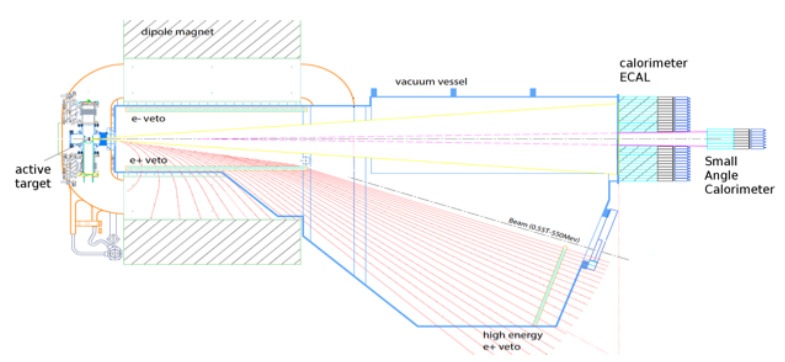

Figure 3. Layout of the PADME detector.

The collaboration aims to complete the construction of the experiment in early 2018 and to collect $\sim 10^{13}$ positrons on target by the end of 2018. The data taking is scheduled to start by April 2018. PADME experiment at LNF-INFN, shown in Fig. 3 will use $550 \mathrm{MeV}$ positron impinging on $100 \mu \mathrm{m}$ thick active target made of polycrystalline diamond. The recoil photons from $e^{+} e^{-} \rightarrow \gamma A^{\prime}$ annihilation process will be detected by a quasi-cylindrical BGO crystal calorimeter made of $61620 \times 20 \times 230 \mathrm{~mm}^{3}$ crystals, located $3.3 \mathrm{~m}$ downstream the target, providing very precise energy and position measurements. The non-interacted beam positrons, will be deflected outside the acceptance of the calorimeter by a $1 \mathrm{~m}$ long dipole magnet. Three different sets of plastic scintillator bars, two of which located inside the magnet gap, will serve to detect the charged particles, thus providing an efficient veto for for the positron bremsstrahlung background. In addition, a very fast small angle calorimeter will be placed along the beam axis, to help with the suppression of the three photon annihilation background. The entire setup will be located in vacuum to minimise the possible beam-residual gas interactions.

\subsection{The PADME detector status}

The construction of the PADME experiment is expected to be completed in early 2018 and the preparation of the different detectors proceeds according to the schedule. In Fig. 4 some pictures of the prototypes used to asses the detector performance are collected.

The $100 \mu \mathrm{m}$ active diamond target with graphite strips readout[16] was one of the most challenging parts of the PADME experiment. After several test on the BTF beam line in Frascati [17] the detector construction has been successfully completed and the in vacuum support system tested. 
The PADME dipole magnet, an MBP-S dipole of the SPS transfer lines, providing $0.55 \mathrm{~T}$ magnetic field over a large $\left(200 \times 500 \times 1000 \mathrm{~mm}^{3}\right)$ gap, has been characterised at Laboratori Nazionali di Frascati and the mechanical support for the integration is in construction.
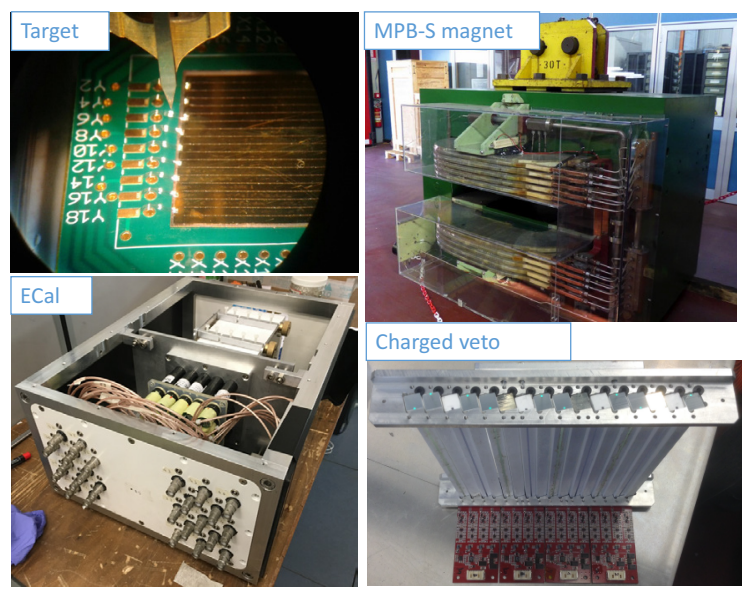

Figure 4. Prototypes of the PADME detector.

The prototypes of the electromagnetic calorimeters and charged veto detector systems have been finalised and tested using electrons and positrons from the LNF Beam Test Facility [18]. Encouraging performance, in agreement with previous L3 results obtained with BGO crystals, have been achieved with the calorimeter prototype[19]. All the BGO crystals and plastic scintillator bars needed for the full detector construction are currently under test to be ready for final assembly.

The PADME experiment readout is based on the CAEN V1742 digitiser board (1-5 Gs/s and 12bit ADC) with optical link readout[20]. About 1000 channels system including the readout of the electromagnetic calorimeter, SAC, and charged particle veto sub-detectors is already available and under test using the prototype data acquisition software. A prototypes of the Level 0 trigger distribution system has been successfully tested achieving the required synchronisation performance. The running of the experiment will start in April 2018 with the commissioning of the PADME beam line.

\subsection{The PADME experiment sensitivity}

The PADME experiment sensitivity estimate is based on a GEANT4 simulation extrapolated to $10^{13}$ positrons on target (POT). This number of particles can be obtained by running PADME for 2 years at $50 \%$ efficiency with 5000 $\mathrm{e}^{+}$per $40 \mathrm{~ns}$ bunch at a repetition rate of $49 \mathrm{~Hz}$. Recent upgrade of the DAФNE LINAC gun allows to extend the bunch length up to $200 \mathrm{~ns}$, when the LINAC is operated in dedicated mode. In this conditions a data sample of $1 \times 10^{13}$ POT can be obtained in 6 month while in 2 years a sample of $4 \times 10^{13}$ POT is within reach. The bounds obtained for $A^{\prime}$ decaying into invisible particles are shown in Fig. 5. The red line shows the expected bound for $1 \times 10^{13}$ POT while the red dashed the one for $4 \times 10^{13}$ POT. The favoured (g-2) $\mu$ region can be explored in a model independent way (the only hypothesis is that $A^{\prime}$ couples to leptons) up to masses of $23.7 \mathrm{MeV}$ [15] . Exploiting the capability of detecting photon and charged particles, the PADME experiment will be able to explore other type of dark sectors mediators such as Axion Like Particles, protophobic X boson[21], and Dark Higgs.

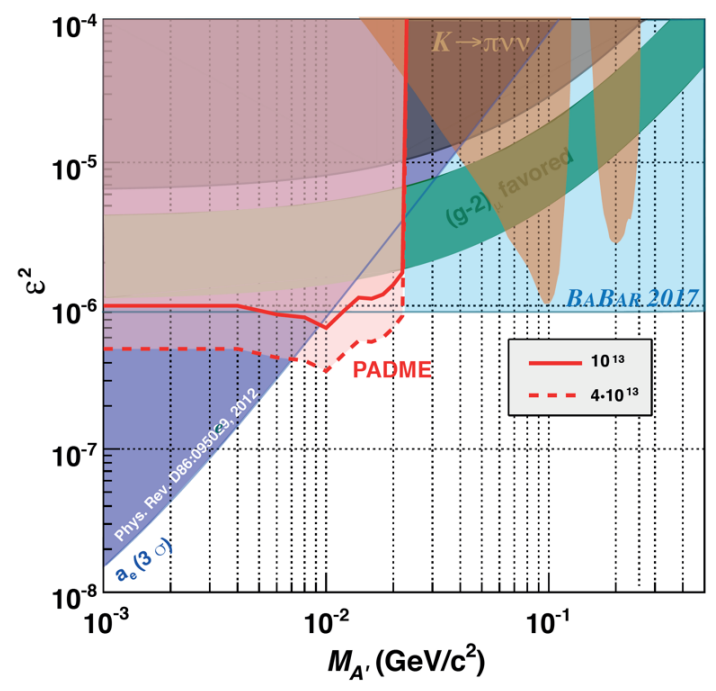

Figure 5. PADME estimated sensitivity for $A^{\prime}$ decaying into invisible particles for different values of the integrated luminosity.

\section{Conclusions}

Dark sectors models offer a simple and phenomenological rich explanation for different Standard Model anomalies, together with a natural solution to the dark matter problem. The existence of new mediators, or dark matter particles, can be probed at low energy accelerators using existing precision experiments or with new dedicated detectors able to explore the intensity frontier. In the next 2-5 years, several new experimental results are expected to enlighten our knowledge on the nature of dark matter.

\section{References}

[1] M. Battaglieri et al., arXiv:1707.04591 [hep-ph].

[2] M. Raggi and V. Kozhuharov, Riv. Nuovo Cim. 38, no. 10, 449 (2015).

[3] J. R. Batley et al. [NA48/2 Collaboration], Phys. Lett. B 746, 178 (2015)

[4] H. Merkel et al., Phys. Rev. Lett. 112, no. 22, 221802 (2014)

[5] J. P. Lees et al. [BaBar Collaboration], Phys. Rev. Lett. 113, no. 20, 201801 (2014)

[6] J. D. Bjorken et al., Phys. Rev. D 38, 3375 (1988).

[7] J. P. Lees et al. [BaBar Collaboration], Phys. Rev. Lett. 119, no. 13, 131804 (2017)

[8] D. Banerjee et al. [NA64 Collaboration], Phys. Rev. Lett. 118, no. 1, 011802 (2017) 
[9] E. Cortina Gil et al. [NA62 Collaboration], JINST 12, no. 05, P05025 (2017)

[10] T. Yamanaka [KOTO Collaboration], PTEP 2012, 02B006 (2012). doi:10.1093/ptep/pts057

[11] J. R. Batley et al. [NA48/2 Collaboration], Phys. Lett. B 769, 67 (2017)

[12] C. Y. Chen, M. Pospelov and Y. M. Zhong, Phys. Rev. D 95, no. 11, 115005 (2017)

[13] R. Marchevski et al. [NA48/2 Collaboration], PoS ICHEP 2016, 636 (2016).

[14] M. Raggi and V. Kozhuharov, Adv. High Energy Phys. 2014, 959802 (2014)

[15] M. Raggi, V. Kozhuharov and P. Valente, EPJ Web Conf. 96, 01025 (2015)
[16] M. De Feudis et al., Nuovo Cim. C 39, no. 1, 254 (2016). doi:10.1393/ncc/i2016-16254-5

[17] R. Assiro et al., arXiv:1709.07081 [physics.ins-det].

[18] P. Valente et al., arXiv:1603.05651 [physics.acc-ph].

[19] M. Raggi et al., Nucl. Instrum. Meth. A 862, 31 (2017)

[20] E. Leonardi, M. Raggi and P. Valente, J. Phys. Conf. Ser. 898, no. 3, 032024 (2017).

[21] J. L. Feng, B. Fornal, I. Galon, S. Gardner, J. Smolinsky, T. M. P. Tait and P. Tanedo, Phys. Rev. D 95, no. 3, 035017 (2017) 